\title{
Un método para la evaluación y auto-optimización de la capacidad de mentalizar del terapeuta, en su trabajo clínico
}

\author{
Gustavo Lanza Castelli ${ }^{1, i}$ \\ Buenos Aires, R. Argentina
}

\begin{abstract}
El trabajo comienza reseñando una investigación que muestra que cuanto mayor es la capacidad de mentalizar del terapeuta, mejores son los resultados de su práctica clínica. Refiere un método utilizado en Canadá para optimizar la mentalización de terapeutas que están haciendo su formación. A continuación desarrolla el método propuesto, que puede ser puesto en práctica por parte del terapeuta en su trabajo clínico, independientemente de su marco teórico de referencia. Dicho método consta de 3 partes: 1) El punto de vista del terapeuta, 2) Las conjeturas del terapeuta sobre el punto de vista del paciente, 3) El punto de vista del paciente. En las dos primeras partes el terapeuta se formula una serie de preguntas que debe responder por escrito, que buscan incrementar su reflexividad. En la tercera, se le pide al paciente que responda a las mismas preguntas, a los efectos de poder contrastar las respuestas del paciente con las conjeturas del terapeuta.

No obstante, es posible utilizar sólo las partes 1) y 2), sin involucrar al paciente en la experiencia. Se enumeran los beneficios de cada una de las partes para la optimización de la capacidad de mentalizar y se ilustra el proceso con un material clínico.

Palabras clave: mentalización del terapeuta - auto-optimización del mentalizar del terapeuta - método para la auto-optimización del mentalizar - eficacia clínica según el mentalizar.
\end{abstract}

The present paper begins by summarizing an investigation which shows that the higher the capacity for mentalizing the therapist has, the better the results in his clinical practice. This work also refers a method used in Canada to improve mentalization in training therapists and develops the proposed method, which can be put into practice regardless his theoretical background. This method consists of 3 parts: 1 ) the therapist's point of view, 2) the therapist's hypotheses on the patient's point of view, 3) the patient's point of view. In the first two parts the therapist makes himself a number of questions to be answered in writing, aiming at an increase in his reflexivity. In the third part, the patient is asked to answer the same questions so that his answers can be contrasted to the therapist's hypotheses. However, it is posible to use only parts 1) and 2), not involving the patient in the experience. The benefits of each of these parts are explained and the process is illustrated with a clinical case.

Key Words: therapist mentalization- self optimization of the therapist mentalization- method for the selfootimization of mentalizing- clinical efficacy depending on mentalization

English Title: A method for the assessment and self-optimization of the therapist's capacity for mentalizing in his clinical work

\section{Cita bibliográfica / Reference citation:}

Lanza Castelli, G. (2017). Un método para la evaluación y auto-optimización de la capacidad de mentalizar del terapeuta, en su trabajo clínico. Clínica e Investigación Relacional, 11 (3): 626-651. [ISSN 1988-2939] [Recuperado de www.ceir.info ] DOI: 10.21110/19882939.2017.110311

\footnotetext{
${ }^{1}$ Psicoanalista. Psicoterapeuta acreditado por la Federación Latinoamericana de Psicoterapia y el World Council for Psychotherapy. Presidente de la Asociación Internacional para el Estudio y Desarrollo de la Mentalización. Ex profesor titular de la materia Clínica Psicológica y Psicoterapias en la carrera de Psicología de la Universidad de Ciencias Empresariales y Sociales. Ex profesor titular de la materia Patologías del Narcisismo del postgrado de especialización en Psicoanálisis, de la Asociación de Psicólogos de Buenos Aires. Fue Secretario del Congreso Mundial de Psicoterapia que tuvo lugar en Buenos Aires, en el año 2005. Director de Mentalización. Revista de Psicoanálisis y Psicoterapia (http://revistamentalizacion.com/). Véase información sobre sus publicaciones en la nota final. Correo electrónico: gustavo.lanza.castelli@gmail.com Página web: $\underline{\text { www.mentalizacion.com.ar }}$
}

CeIR Vol. 11 (3) - Octubre 2017 ISSN 1988-2939 - www.ceir.info

(c) Derechos reservados/Copyright de Clínica e investigación Relacional y los autores. Prohibida la reproducción total o parcial sin autorización expresa. Este material es para uso científico y profesional exclusivamente y puede contener información clínica sensible. Los editores no se responsabilizan de los contenidos de los autores. Dirigir las consultas sobre derechos y autorizaciones a ceir@psicoterapiarelacional.es 
Las investigaciones que han tomado como objeto al terapeuta para determinar cuáles son las variables que le pertenecen, responsables de mejores resultados terapéuticos, no han arrojado hasta el momento resultados significativos, en la medida en que han tomado en consideración el género del terapeuta, su formación, marco teórico, experiencia, etc. Ninguna de esas variables ha demostrado marcar una diferencia significativa en cuanto a la efectividad del quehacer de los profesionales (Baldwin \& Imel 2013; Lambert 2013).

Pero una investigación reciente arroja un resultado diferente, en tanto muestra que cuanto mayor es la capacidad de mentalizar del terapeuta, mejores son los resultados clínicos. La variable "capacidad de mentalizar" (o nivel de la Función Reflexiva) sí parece marcar una diferencia apreciable en los resultados clínicos que logran terapeutas que poseen un alto grado de FR.

La investigación empírica a la que me refiero, fue publicada en enero de 2017, y llevada a cabo por Cologon, Schweitzer, King y Nolte, quienes evaluaron el desempeño de 25 terapeutas, que trataron a 1.000 pacientes, en cuanto a los resultados obtenidos.

Asimismo, evaluaron también cuidadosamente el nivel de FR de los terapeutas.

Encontraron que las diferencias en los resultados no podían explicarse por diferencias en la experiencia de los terapeutas, en su formación o en su training, o en función de otras variables de personalidad. Tampoco por su enfoque teórico, que era en lo esencial de corte psicodinámico en todos los casos.

Para indagar la incidencia del nivel de FR en los resultados que lograban, los dividieron en 3 grupos, en función de su capacidad para mentalizar, que evaluaron mediante la entrevista de apego adulto, interpretada con la escala de Fonagy et al. (1998). De este modo se formó un grupo en el que los terapeutas tenían un elevado nivel, otro en el que tenían un nivel medio y un tercero en el que el nivel era bajo.

Los terapeutas pertenecientes al grupo con mentalización superior (mayor que la media de la población) fueron los que lograron mejores resultados. Sus pacientes fueron los que tuvieron mayor reducción en su sintomatología.

Los pacientes de los terapeutas con un nivel mentalizador igual al término medio de la población, mejoraron también, pero en menor medida y necesitando más tiempo para alcanzar dicha mejoría.

Los pacientes de los terapeutas cuya capacidad mentalizadora era baja, menor que la media, no lograron mejorías significativas. 
De este modo, se comprobó que la diferencia en la efectividad de los terapeutas, correlacionaba con su capacidad para mentalizar (Cologon et al., 2017).

Este hallazgo tiene, sin duda, importantes consecuencias prácticas. Una de ellas es que puede poseer la mayor utilidad evaluar la capacidad mentalizadora de los aspirantes a terapeutas, de modo tal que aquellos que posean una capacidad baja, lleven a cabo un entrenamiento que les ayude a optimizarla. Dando un paso más en esa dirección, cabe postular que dicho entrenamiento podría formar parte de la capacitación de todo aquel que aspire a desempeñarse como terapeuta.

En lo que sigue, reseño brevemente un método que se utiliza para este fin y, posteriormente, caracterizo un método que he diseñado -al que denomino "Método de Auto-optimización de la Capacidad de Mentalizar"- mediante el cual es posible llevar a cabo, en forma personal, un entrenamiento que permite que el terapeuta (novel o ya formado) evalúe constantemente su capacidad de mentalizar, tanto respecto de sí mismo en su trabajo, como respecto de tal o cual paciente determinado, y la optimice a lo largo del período de tiempo que él considere útil o necesario.

De igual forma, procura que el profesional posea herramientas para identificar en qué punto ha fallado, así como para anoticiarse de las razones de sus fallos eventuales, de modo tal que dicha identificación le sirva para el autoanálisis de los motivos de los mismos, su inclusión en la supervisión, o aún su discusión en el interior de su análisis personal.

Dicho método tiene tres partes, que pueden denominarse de la siguiente forma:

1) El punto de vista del terapeuta.

2) Las conjeturas del terapeuta sobre el punto de vista del paciente

3) El punto de vista del paciente y su contrastación con las conjeturas del terapeuta.

Si bien el método completo incluye las tres partes a los efectos de obtener el máximo aprovechamiento del mismo, es posible llevarlo a cabo parcialmente, mediante la realización de solamente las partes 1) y 2), sin inclusión de la tercera.

En lo que sigue, intento caracterizar de un modo más preciso qué podemos entender por capacidad de mentalizar del terapeuta, describo un entrenamiento utilizado en Canadá para favorecer el incremento de dicha capacidad y, posteriormente, caracterizo el método que propongo y lo ilustro con un ejemplo personal. 


\section{La capacidad de mentalizar del terapeuta:}

Podríamos decir que en principio esta capacidad ha de homologarse sin más con la capacidad de mentalizar, la cual cabe definir sucintamente como la capacidad para comprender el comportamiento propio y ajeno en términos de estados mentales, regular la propia experiencia emocional y establecer relaciones interpersonales que tengan en cuenta la mente propia y la ajena, así como su interacción.

Ampliando un poco esta definición tan escueta, podríamos decir que en tanto consideramos a dicho constructo como multidimensional, diferenciamos en él cuatro polaridades: mentalización del self/mentalización del otro; mentalización automática o implicita/mentalización controlada o explícita; a predominio cognitivo/a predominio afectivo; centrada en lo interno/centrada en lo externo (Fonagy, Luyten, 2009, 2010).

A su vez, cabría agregar que podemos diferenciar cuatro dominios en la capacidad de mentalizar: 1) la capacidad para comprender la naturaleza de los estados mentales, 2) la capacidad para comprender la mente propia; 3) la capacidad para comprender la mente ajena; 4) las capacidades de regulación, atencional, emocional y conductual (Lanza Castelli, Bilbao Bilbao, 2012).

De todos modos, en lo que hace a la capacidad de mentalizar del terapeuta, es posible agregar que hay ciertas capacidades del mentalizar que se revelan como particularmente importantes para su quehacer, independientemente de su marco teórico de referencia. Algunas de ellas son:

a) la capacidad para aprehender -de un modo descentrado, esto es, desde el punto de vista del paciente- los estados mentales de este último en toda su complejidad, así como las relaciones que se establecen entre ellos (formación de conglomerados, oposición y conflicto, secuencias y desenlaces, etc.) y el modo en que se manifiestan en sus vínculos y en su conducta. Esta capacidad incluye la aprehensión de los estados mentales inconscientes (Fonagy et al., 2002).

b) la habilidad para evaluar el momento adecuado para intervenir, así como la forma de hacerlo, y el poder anticipar cómo habrá de impactar dicha comunicación en el paciente.

c) la capacidad para registrar los propios procesos de contra-transferencia.

d) la habilidad para conectarse con las propias ocurrencias y asociaciones, y relacionarlas con el decir del paciente, así como con la propia y personal problemática, estableciendo la diferencia entre una y otra alternativas, etc.

e) otra particularidad del mentalizar del terapeuta, es que éste no "se deja ir" en el flujo de la interacción, guiado simplemente por la mentalización automática, como solemos hacer en relaciones personales de afecto y confianza, sino que monitorea constantemente, evalúa y 
controla sus reacciones y exteriorizaciones espontáneas, en función de la pertinencia, o de la falta de ella, en cuanto a traducirlas sin más en verbalizaciones (interpretativas o de otra índole). Esto supone un ir y venir de la mentalización automática a la controlada, que no es habitual en las interacciones cotidianas.

Este listado, seguramente incompleto, nos muestra no obstante, que hay determinadas facetas de la mentalización que no son muy frecuentes en el mentalizar de la vida cotidiana, pero que son necesarias en el mentalizar del terapeuta, por lo cual las exigencias son mayores para este último.

\section{Entrenamiento para optimizar la capacidad de mentalizar del terapeuta}

Un grupo de profesionales en Canadá ha desarrollado un training específico en mentalización para terapeutas noveles, que han de trabajar con pacientes difíciles de comprender, como son los TLP.

En un artículo en el que dan cuenta de su proceder, estos colegas relatan que llevaron a cabo una investigación con 40 terapeutas noveles, que no tenían todavía experiencia clínica, a los que les presentaron viñetas de pacientes TLP (Ensink et al., 2013).

Los terapeutas fueron divididos en dos grupos. El primero de ellos (grupo control) recibió el entrenamiento habitual, que incluía conocimientos sobre psicopatología, teoría, DSM, etc.

El segundo grupo recibió -además de esos conocimientos- un entrenamiento especial destinado a incrementar su capacidad de mentalizar, que tenía un carácter experiencial y que estimulaba a los estudiantes a que utilizaran sus reacciones personales para comprender mejor a los pacientes, diferenciaran distintas formas en que trabajaba su propia mente, identificaran lo que les hacía obstáculo para comprender el material clínico, hablaran de sus propias reacciones emocionales y discutieran material didáctico sobre mentalización, que aplicaban a ejemplos clínicos.

Los resultados mostraron que dicha capacidad se incrementó en el segundo grupo, pero no en el primero, y que los participantes del grupo que incluyó el training en mentalización estuvieron en mejores condiciones de comprender lo mostrado en las viñetas, que aquellos pertenecientes al primer grupo.

En uno de los pasajes de su trabajo, los autores expresan lo siguiente: "El entrenamiento en mentalización produjo un incremento de aproximadamente un punto (de 1.2 a 2) en una escala de 5 puntos utilizada para evaluar la FR de los terapeutas. En términos prácticos, representa un incremento desde una comprensión rudimentaria hasta una comprensión básicamente buena, 
lo que parece un objetivo deseable y realista. Por otra parte, en el grupo control no se observó un incremento en la FR (...) Esto indica que, a menos que la FR sea tomada específicamente como objeto, de modo tal que se realice un esfuerzo para desarrollar esta habilidad, ella no se desarrolla espontáneamente" [negritas agregadas] (p. 19)

Esta última idea resulta sumamente interesante y, si se la toma seriamente, hace pensar que, en efecto, algo ha de hacerse para desarrollar la capacidad de mentalizar, ya que ésta correlaciona en forma directa con la mejoría que experimenten, o no, nuestros pacientes (sin dejar de lado, de más está decirlo, la necesidad de la formación, la supervisión y el análisis personal), según muestra la primera investigación mencionada

Es indudable que el entrenamiento en mentalización propuesto por los colegas canadienses resulta sumamente atractivo y sería de desear que se expandiera a los más variados institutos de formación de terapeutas.

No obstante, considero que aún teniendo en cuenta lo valioso que resulta el training mencionado, también es posible llevar a cabo, en forma personal, un entrenamiento que permita que el terapeuta (novel o ya formado) evalúe constantemente su capacidad de mentalizar, tanto consigo mismo como con tal o cual paciente, y la optimice a lo largo del período de tiempo que él considere útil o necesario.

\section{Caracterización del Método de Auto-optimización de la Capacidad de Mentalizar}

Como fue dicho con anterioridad, el método que he diseñado tiene tres partes, que pueden caracterizarse de la siguiente forma:

1) El punto de vista del terapeuta: esta parte del método consiste en que el terapeuta se plantee a sí mismo una serie de preguntas sobre alguna sesión que haya tenido lugar (preferentemente en ese mismo día) e intente responderlas del modo más claro y exhaustivo que le sea posible.

Dichas preguntas, son las siguientes:

a) ¿Qué fue para mí lo más importante de la sesión? (¿fue algo que pasó, que fue dicho, que el/la paciente sintió, pensó o hizo?).

¿Por qué fue lo más importante?

¿En qué me baso para suponer esto? ¿Qué indicadores tomo en cuenta?

b) ¿Cómo evalúo mi actitud para con él/ella y mis intervenciones?

¿En qué baso mi evaluación? ¿Qué indicadores tomo en cuenta?

c) ¿Cuál de mis intervenciones creo que fue la más útil y cuál creo que fue la menos útil? 
¿En qué me baso para suponer esto? ¿Qué indicadores tomo en cuenta?

Estas preguntas están dirigidas a estimular la mentalización del terapeuta sobre sí mismo (mentalización del self), e incluyen ya (pregunta c) un comienzo de mentalización del otro (Cf. 2 da. parte). En este sentido, la pregunta por los indicadores favorece que éste pueda explicitar y poner de manifiesto para sí mismo su propio funcionamiento intuitivo (procedimental, mentalización implícita) y/o dar forma verbal a impresiones sensoriales, visuales, cenestésicas, a pensamientos incipientes que, de no ser atendidos y verbalizados permanecen muchas veces sin forma en el interior de nuestra psique, lo que dificulta su percepción (Lanza Castelli, 2010b). El poner en palabras transforma dichos contenidos, así como el funcionamiento intuitivo, en conocimiento declarativo (mentalización explícita) pasible de evaluación, contrastación y modificación.

Cabe agregar en este punto, que la mentalización implícita no es reflexiva ni consciente, tiene un tiempo de procesamiento rápido, procesa estímulos en paralelo (gestos, tonos de voz, ritmo del habla, posturas, etc.), requiere poco esfuerzo, atención concentrada o intención y suele ser caracterizada como intuición (Allen, Fonagy, Bateman, 2008).

Por esta razón, requiere un considerable esfuerzo muchas veces, llegar a traducir estas impresiones rápidas, preconscientes y complejas, en mentalización explícita, que se basa en las palabras, es consciente, deliberada y reflexiva, tiene un procesamiento serial y lento, supone atención concentrada y esfuerzo.

No obstante, cuando se logra esta "traducción" el terapeuta se ha enterado mejor de sus procesos involuntarios, ha identificado con mayor claridad sus intuiciones y el por qué de las mismas, ha dado forma a las sensaciones y fragmentos de pensamiento mencionados y los ha percibido con mayor claridad, lo cual implica que ha conquistado un grado mayor de mentalización.

Al responder a las preguntas por los indicadores, el terapeuta puede tomar mayor conciencia de si llega a sus conclusiones intuitivamente (mentalización implícita), o si las mismas se basan en indicadores elocuentes del paciente, que aprehendió de un modo reflexivo (mentalización deliberada), o se basan en postulados teóricos, o en reflexiones y razonamientos, etc., lo que le ayuda a aprehender cómo funciona su propia mente durante su trabajo clínico. Esta aprehensión puede serle de utilidad para corregir eventuales unilateralidades en sus procedimientos, enriqueciendo de esta forma su funcionamiento mental durante la sesión. 
2) Las conjeturas del terapeuta sobre el punto de vista del paciente:

En esta segunda parte, el terapeuta ha de cambiar su posición. Ya no se focalizará en sí mismo (mentalización del self), sino que intentará centrarse en lo experimentado por el paciente, tal y como él lo ha podido entender (mentalización del otro).

Se formulará entonces, las siguientes preguntas, que responderá también por escrito:

a) ¿Qué fue para el/la paciente lo más importante de la sesión? (¿Fue algo que pasó,

algo que fue dicho, algo que sintió, o que pensó, o que hizo?)

¿Por qué para él/ella fue lo más importante?

¿En qué me baso para suponer esto? ¿Qué indicadores tomo en cuenta?

b) ¿Cómo se sintió a lo largo de la sesión? (Mencionar por lo menos dos

momentos de la sesión y lo que sintió)

¿En qué me baso para suponer esto? ¿Qué indicadores tomo en cuenta?

c) ¿Cómo sintió mi actitud para con él/ella y mis intervenciones?

¿En qué me baso para suponer esto? ¿Qué indicadores tomo en cuenta?

d) ¿Cuál de mis intervenciones fue la que -desde el punto de vista de él/ella- le resultó más útil y cuál la que le resultó menos útil?

¿En qué me baso para suponer esto? ¿Qué indicadores tomo en cuenta?

Como podemos ver, en esta segunda parte se repiten las mismas preguntas que en la primera y se agrega una cuarta pregunta, la número b), que no estaba presente en la primera parte, pero que se repetirá en la tercera.

La diferencia fundamental con la parte anterior es que ahora dichas preguntas intentan promover que el terapeuta se ponga en el punto de vista del paciente (tal como él puede llegar a comprenderlo) e intente responder desde ahí a dichas preguntas, lo que es un procedimiento diferente a responderlas desde sí mismo, que exige procesos mentalizadores también distintos. Entre estos procesos los fundamentales son: la inhibición de la propia perspectiva para poder aprehender la perspectiva ajena (Allen, Fonagy, Bateman, 2008), el descentramiento, el ponerse en el punto de vista del otro intentando aprehender desde su interior el modo en que ha vivido lo formulado en las preguntas.

De la mayor importancia resulta el hecho de que al responder a estas preguntas el terapeuta puede advertir mejor si lo que piensa que ha experimentado el paciente es lo que él mismo había pensado de los aspectos de la sesión mencionados en las preguntas de la primera parte. 
Por ejemplo, si lo que él supone que ha sido importante de la sesión para el paciente, es lo que él mismo consideró importante de dicha sesión. En ese caso cabe la posibilidad de que le esté atribuyendo al paciente su propio proceso mental. La alternativa opuesta tiene lugar cuando el terapeuta puede mencionar indicadores del paciente (expresiones, verbalizaciones, tonos de voz, gestos, movimientos, etc.), que lo llevan a considerar que ese pasaje de la sesión fue el más importante para el consultante. No es infrecuente una conjunción de ambas alternativas.

La reiteración de esta práctica posee la mayor utilidad para el desarrollo del aprendizaje sobre el propio modo de entender al paciente, que tiene que ver con la mentalización del otro (y es distinto del autoanálisis dirigido a los propios conflictos).

En el aspecto práctico, suele resultar útil que el terapeuta escriba en primer término la primera parte, esto es, qué le pareció a él importante, qué intervenciones cree que fueron útiles, etc. Una vez hecho esto, ha de llenar los ítems en los que consigna lo que supone que experimentó el paciente.

El escribir previamente cómo se sintió él, puede servirle para objetivarlo y advertir mejor, cuando escribe sobre el paciente, si simplemente está proyectando su sentir, o si está aprehendiendo (o intentando aprehender) cuál ha sido la experiencia del paciente. Dicho de otra manera: al escribir primero sobre sí mismo, puede tomar conciencia de las dificultades que encuentra para descentrarse, y lograr, eventualmente, un mayor descentramiento.

Algunos de los rendimientos que es posible obtener mediante la puesta en acto de esta segunda parte, serán mencionados en el ejemplo clínico y en las consideraciones finales.

\section{3) El punto de vista del paciente y su contrastación con el punto de vista del terapeuta}

Esta tercera parte cambia radicalmente el eje presente en la segunda parte, en la medida en que no se trata ya de lo que el terapeuta conjetura o imagina que experimentó el paciente desde su propio punto de vista, sino de la inclusión en la escena del punto de vista del paciente, del que nos enteramos en tanto le preguntamos por él.

Las preguntas que se le hacen al paciente, se le dicen verbalmente y se le entregan también por escrito, solicitándole que las responda de ser posible ese mismo día, o al día siguiente, y que las envíe por mail al terapeuta, o las lleve a la sesión siguiente para trabajar sobre lo que escribió, si tal cosa fuera deseable o necesaria.

En un trabajo anterior he denominado a este procedimiento "diario de sesiones" y he intentado mostrar su utilidad para favorecer el incremento del mentalizar en el paciente, para brindar un feedback al terapeuta y para una serie de rendimientos que sería demasiado extenso 
desarrollar nuevamente en este lugar (Lanza Castelli, 2008). Por esa razón, si bien incluiré en lo que sigue las preguntas que se le entregan al paciente, no haré comentario alguno sobre la utilidad que tienen para éste, sino sobre el trabajo que el terapeuta lleva a cabo a partir de la contrastación de sus propias conjeturas con las respuestas del consultante.

Supongamos un paciente al que llamaremos Carlos. Se le entregará el siguiente texto:

Carlos, te pido que pongas por escrito (y me envíes por mail):

a) ¿Qué fue para ti lo más importante de la sesión? (algo que pasó, algo que

fue dicho, algo que sentiste, o que pensaste, o que hiciste).

¿Por qué fue lo más importante?

b) ¿Cómo te sentiste a lo largo de la sesión? (Menciona por lo menos dos

momentos de la sesión y describe cómo te sentiste)

c) ¿Cómo sentiste mi actitud para contigo y mis intervenciones?

d) ¿Cuál de mis intervenciones fue la que te resultó más útil y cuál la que te resultó menos útil?

Una vez que el terapeuta ha escrito su parte y recibe el material del paciente, procede a contrastarlos. Establece entonces las concordancias y las discrepancias, esto es, busca establecer hasta qué punto lo que él supuso que, por ejemplo, para el paciente había sido lo más importante de lo ocurrido en la sesión, ha sido efectivamente tal como él creyó percibirlo, o si, por el contrario, hay una diferencia apreciable entre su conjetura y lo vivido por el paciente, lo que hablaría de una falla en su capacidad de mentalizar, en ese punto concreto. $Y$ otro tanto cabe decir respecto a las otras preguntas.

Las discrepancias detectadas por el profesional pueden iniciar un segundo circuito interrogativo, en el cual el terapeuta volverá sobre su anotación errónea, tratando de reconstruir con el mayor detalle que le sea posible los motivos que lo llevaron a escribir esa respuesta. De este modo es posible que descubra un sesgo interpretativo que lo lleva a otorgar un sentido particular a determinadas verbalizaciones o exteriorizaciones del paciente, como así también puntos ciegos, dificultades para captar determinada gama de sentimientos o conflictos, etc.

También es posible que estos descubrimientos sean el punto de partida de un autoanálisis, que lo lleve a descubrir las razones de estas limitaciones, en diversos factores de su personalidad. 
La reiteración de dichas fallas podrá inclinarlo a llevarlas a supervisión o a su análisis personal, ya que en ese caso es posible que esté incidiendo una variable que el terapeuta no puede aprehender por sí mismo.

El método que estoy proponiendo le permite al terapeuta, como hemos dicho ya, evaluar su capacidad y competencia con tal o cual paciente en particular. Pero también le permite evaluar cómo la misma se va modificando (o no) a medida que avanza el tratamiento y que continúa utilizando el método.

\section{Ejemplo clínico:}

Deseo ahora ilustrar lo mencionado hasta este punto, con un ejemplo de mi práctica clínica, consistente en lo escrito en un momento puntual del tratamiento de una paciente fronteriza, particularmente difícil y polisomatizante. Su compleja historia de vida, así como una serie de características de su personalidad y de los avatares del tratamiento, poseen el mayor interés y merecerían un trabajo en sí mismos, pero en este caso he de abreviar al máximo dicha información, para no desviarnos del foco de este escrito.

Baste decir que la llamaremos Violeta, que consultó teniendo 33 años llevada por la angustia que le produjo el tener que operarse del intestino, en lo que fue su sexta operación en tres años. A ello se sumaban diversos conflictos de pareja, tendencia a la promiscuidad, situaciones de blanco mental y una proclividad a volverse adicta a su pareja de turno.

En la sesión previa al material escrito que presentaré, había estado hablando de Manuel, hombre con el que salía, pero que no planteaba con claridad la relación que había entre ellos, ni ella se animaba a preguntarle al respecto.

Relató distintas situaciones vividas con él en el fin de semana, a partir de lo cual le señalé que la inseguridad de que la amasen la llevaba a entablare relaciones de dependencia en las que estaba siempre con temor a que la echaran. Asintió a este comentario diciendo que eso la llevaba a no poder ser nunca ella misma cuando estaba en pareja. Entre otras cosas, no se animaba a preguntarle a Manuel si deseaba casarse con ella, o formar una pareja estable y convivir.

Le señalé también que había llegado a la sesión en estado de desconexión y que se había ido conectando a medida que ésta transcurría. Por su parte, aludió en una oportunidad, un poco al pasar, a los estados de vacío en los que entraba en ocasiones y que le producían mucha angustia. En ese momento intenté indagar con más detenimiento en dichos estados, pero Violeta cambió de tema instantáneamente y comenzó a hablar de un modo más desafectivizado. 
Una vez terminada la sesión, me dispuse a aplicar el Método, cosa que hice del siguiente modo:

\section{Aplicación del Método para la Auto-optimización de la Capacidad de Mentalizar}

1) El punto de vista del terapeuta:

a) ¿Qué fue para mí lo más importante de la sesión? (fue algo que pasó, algo que fue dicho, algo que Violeta sintió, o pensó, o que hizo?)

Lo que para mí fue importante de la sesión fue haber podido llevarla a conectarse de nuevo con lo que habíamos dicho la vez pasada. O haberle mostrado su desconexión.

También el haberle mostrado que establece una relación de dependencia con los hombres (ahora me viene a la mente que es una mujer muy superficial, esto pensado con un tono un tanto peyorativo).

¿Por qué fue lo más importante?

Porque considero que para ella es fundamental ir removiendo ese mecanismo de desconexión e ir conectándose con el vacío y el desamparo interior, respecto de los cuales utiliza como defensa la exaltación (como la que tiene ahora con Manuel).

¿En qué me baso para suponer esto? ¿Qué indicadores tomo en cuenta?

Todo hasta ahora me indica que éste es un tema clave en su vida, responsable de muchos de sus síntomas y cuyo origen en la temprana relación con la madre será importante poder desentrañar y elaborar.

Los indicadores que tomo en cuenta son sus propios relatos acerca de las situaciones de desconexión y las veces que esto sucede en el transcurso de la sesión.

b) ¿Cómo evalúo mi actitud para con ella y mis intervenciones?

Creo que pude mantenerme conectado todo el tiempo, muy atento a las modificaciones en su estado que se iban produciendo. En cuanto a las intervenciones, tal vez en algunas ocasiones actué un poco apresuradamente, ya que me adelanté a lo que ella estaba por decir (¿contratransferencia?).

¿En qué baso mi evaluación? ¿Qué indicadores tomo en cuenta?

En relación a lo primero que puse, el indicador fue la conciencia que tenía de estar plenamente atento, cosa que puedo recordar ahora sin dificultad. En relación a lo segundo, el interrumpirla para hacer mis comentarios.

c) ¿Cuál de mis intervenciones creo que fue la más útil y cuál creo que fue la menos 
útil?

Creo que fue de utilidad la que le hice sobre la desconexión, pero no aquélla en la que intenté explorar los estados de vacío.

¿En qué me baso para suponer esto? ¿Qué indicadores tomo en cuenta?

Su retirada inmediata (cambio de tema) cuando intenté explorar, me hace pensar que no advertí que ése no era un camino transitable.

2) Las conjeturas del terapeuta sobre el punto de vista del paciente: en esta segunda parte respondí de la siguiente forma las preguntas que me formulé:

a) ¿Qué fue para Violeta lo más importante de la sesión? (¿Fue algo que pasó,

algo que fue dicho, algo que sintió, o que pensó, o que hizo?)

Creo que lo más importante para ella fue hablar del estado de desconexión, que yo le mostrara eso. También lo que le dije de la relación de dependencia que ella establece con los hombres.

¿Por qué para ella fue lo más importante?

Porque me dio la impresión que ella veía lo que yo le decía, que le parecía importante en su vida. Más lo primero que lo segundo.

¿En qué me baso para suponer esto? ¿Qué indicadores tomo en cuenta?

Los indicadores que tomo en cuenta son la forma en que ella asentía a lo que le decía y el hecho que poco después de que le comentara esto, comenzó a conectarse cada vez más, al punto que en la parte final de la sesión estaba muy conectada y participativa, siendo que había entrado en estado de desconexión (ahora que escribo esto, me doy cuenta que estoy confundiendo su perspectiva con la mía. Fui yo quien advirtió ese incremento en su conexión y la modificación que tuvo lugar a lo largo de la sesión, pero no sé si ella también lo notó, o simplemente lo fue viviendo, sin considerarlo particularmente importante)

b) ¿Cómo se sintió a lo largo de la sesión? (Mencionar por lo menos dos

momentos de la sesión y lo que sintió).

Creo que se sintió entendida y acompañada por mí en lo que iba relatando.

Un momento significativo fue cuando mencionó su miedo a preguntarle a Manuel si quería casarse con ella. En ese momento se angustió. Otros momentos fueron cuando hablamos de su desconexión y del vacío.

¿En qué me baso para suponer esto? ¿Qué indicadores tomo en cuenta? 
Supongo que se sintió entendida y acompañada porque se fue conectando cada vez más. También porque se involucraba cada vez más. Su tono de voz se fue haciendo más firme, la pose "desgreñada" (Esto no sé cómo denominarlo. Al principio era como que estaba desparramada en el sillón. Después su cuerpo se fue unificando y teniendo una mayor tensión, una mayor vitalidad) se modificó.

Creo que uno de los momentos significativos fue con lo de Manuel y el casamiento, porque me pareció que cuando dijo lo del casamiento se angustiaba. Eso me hace suponer que fue un momento significativo para ella, habida cuenta del enganche que tiene con él.

c) ¿Cómo sintió mi actitud para con ella y mis intervenciones?

Sintió que la entendía, que lo que yo le decía era así, que captaba el motivo por el cual no se anima a preguntarle a Manuel del casamiento, por ej.

¿En qué me baso para suponer esto? ¿Qué indicadores tomo en cuenta?

En que cuando se lo decía, ella decía que sí, pero también porque por momentos, una o dos veces, me adelanté a lo que ella iba a decir, y sonrió al decirlo, como si le gustara que yo me hubiera adelantado, en tanto eso denotaba mi conexión con ella y que la entendía (pero en este punto me siento hipotetizando sin mayores elementos).

d) ¿Cuál de mis intervenciones fue la que -desde el punto de vista de ella- le resultó más útil y cuál la que le resultó menos útil?

La intervención que le resultó más útil fue la referida a su desconexión.

También cuando le comenté la forma en que dependía de los hombres.

¿En qué me baso para suponer esto? ¿Qué indicadores tomo en cuenta?

Porque asintió cuando le hablé de su desconexión y después pudo conectarse. No mencionó que estaba más conectada y que lo que yo le había dicho le había resultado útil, pero en este punto debo considerar que no siempre el paciente nos comenta cómo se va sintiendo con lo que ocurre en la sesión y que es válido inferir que así se sintió

(me acuerdo ahora de las investigaciones de Rennie y de cómo descubrió que los pacientes suelen funcionar en dos planos: uno es el que se explicita ante el terapeuta, el otro es como un río subterráneo, no muy claro para sí mismo, pero rico en pensamientos y sentimientos).

La intervención que le resultó menos útil fue cuando le pregunté por el vacío y cambió de tema, comenzando a hablar de un modo desafectivizado. Creo que no estaba preparada todavía para hablar de eso y que puede haber sentido como perturbadora mi pregunta.

Ahora pienso que ese tema será abordable cuando ella esté más estructurada que lo que 
estaba hoy, y que no pude darme cuenta que ella no estaba en condiciones de hablar de ese asunto..

Pienso también que a mí me resulta muy interesante ese tema y que lo relaciono con los desarrollos de André Green. Supongo que en ese momento primó mi propio interés en explorar ese punto, y de ahí la falla en mi empatía. Veo ahora que es muy importante cuando uno tiene un deseo activado, porque va a incidir en la forma en que encare la sesión, en lo que pregunte (como en este caso), etc. Me parece que ahora entiendo mejor lo que dice Bion, de escuchar "sin memoria y sin deseo" (1970, p. 45), o lo siento más "en carne propia".

Comentarios sobre la primera y segunda partes: aún sin tener todavía el texto de la paciente, podemos reflexionar acerca de algunas de las cosas que tuvieron lugar mientras escribía sobre ella.

Una vez más corroboré lo importante que es escribir en primer término lo que para mí fue importante de la sesión, porque de ese modo es como que quedo aligerado de una tensión y puedo concentrarme mejor en lo que supongo que vivió ella. Además, ya estoy advertido de lo que yo he opinado y entonces es menos probable que se me confunda con lo que supongo de ella.

Para mi sorpresa, cuando estaba escribiendo esta parte apareció el siguiente pensamiento: (ahora me viene a la mente que es una mujer muy superficial, esto pensado con un tono un tanto peyorativo).

En un primer momento lo dejé de lado, pero después volví sobre él, tratando de dejar fluir imágenes y asociaciones...que no tardaron en presentarse. Surgieron varias líneas asociativas, una de las cuales me condujo a un territorio problemático, que prefiero dejar sin revelar aquí. Sólo deseo subrayar que es frecuente que respondiendo las preguntas surjan este tipo de ocurrencias involuntarias, que son de mucha utilidad para el trabajo autoanalítico y para comprender mejor y tratar de elaborar la contratransferencia.

Mi respuesta a la pregunta a) de la segunda parte, coincidente con mi propia impresión sobre lo que había sido importante en la sesión, me hizo dudar por un momento si no se trataba de una proyección. Dicha idea me requirió un esfuerzo extra para recordar las circunstancias en que habíamos hablado del tema y la forma en que ella asentía, lo que me dio a pensar que sí había percibido correctamente.

Cuando hablo de los motivos en que me baso, me llama la atención un deslizamiento nada inhabitual en estos casos: atribuirle a ella algo que había sido observado por mí.

La utilización reiterada de este método me ayudó a darme cuenta de las ocasiones en que incurría en este deslizamiento y a ir neutralizándolo paulatinamente, mediante un 
descentramiento deliberado. En este punto veo un incremento en mi capacidad de mentalizar, que se volvió una adquisición permanente.

En la respuesta a la pregunta b), cuando hablo de los cambios en su actitud corporal, hago consciente y explícito algo que había sido implícito mientras ocurría en la sesión. Me di cuenta con posterioridad que su cambio de actitud había producido que yo estuviera más atento y conectado de lo que ya lo estaba.

En el momento en que escribo se abre ante mí un panorama de vivencias sutiles que había tenido durante la sesión, pero de las que recién al poner por escrito me enteraba.

También en este punto considero que hay un incremento en mi mentalizar, al poder darme cuenta de algo que no había sido previamente consciente, poder ponerlo en palabras y reflexionar sobre mi cambio de actitud (tampoco incluyo en este escrito las conclusiones a las que arribé, ahondando en este punto).

Las respuestas a la pregunta c) no considero que aporten nada de interés en sí mismas. En este punto habrá que ver lo que surge del cotejo con el material de la paciente.

En lo que hace a las respuestas a la pregunta d) hay dos partes. En la primera intento dar cuenta de aquello en lo que me baso y escribo:

"No mencionó que estaba más conectada y que lo que yo le había dicho le había resultado útil, pero en este punto debo considerar que no siempre el paciente nos comenta cómo se va sintiendo con lo que ocurre en la sesión y que es válido inferir que así se sintió (me acuerdo ahora de las investigaciones de Rennie y de cómo descubrió que los pacientes suelen funcionar en dos planos: uno es el que se explicita ante el terapeuta, el otro es como un río subterráneo, no muy claro, pero rico en pensamientos y sentimientos)"

Para fundamentar ante mí mismo en qué me basaba para mi respuesta, argumento que no siempre el paciente nos comenta...etc. En ese momento me vienen a la mente unas muy interesantes investigaciones de David Rennie (1992, 1994, 2001) que había leído años atrás. Este recuerdo me ayudó a comprender mejor lo que estaba queriendo argumentar, por lo cual también en este punto vi más claro.

En la segunda parte, cuando escribo sobre lo que le resultó menos útil, recuerdo mi poco feliz intervención en que le pregunto por el vacío. Gracias a ponerlo por escrito advierto los motivos de mi falla empática (el interés por los desarrollos de Green) y experimento con claridad cómo el propio deseo puede incidir perjudicialmente en las intervenciones que tenemos con los pacientes. Este darme cuenta de lo que me había ocurrido supone también un incremento en el conocimiento de los procesos que tuvieron lugar en mi interior en distintos momentos de la sesión. 
Para finalizar estas consideraciones sobre las partes primera y segunda, querría consignar cómo, aún antes de contrastar mi escrito con el de la paciente, obtuve una serie de rendimientos, uno de los cuales consistió en que lo que había sido una experiencia vivida en el modo que Fonagy y Target (1997) denominaron alguna vez "self prerreflexivo", se transformó en un ejercicio de reflexión sobre mis propios procesos mentales y las actitudes con ellos relacionadas. Vale decir que mi propio proceso de escritura significó un incremento en mi reflexividad y en mi comprensión de mí mismo, en el mentalizar mi propio self, así como en la identificación de ciertas modalidades y problemas en mi comprensión de la paciente.

Volveré sobre este punto al final de este trabajo, en las consideraciones finales y consignaré allí otros rendimientos.

\section{El diario de sesiones de Violeta:}

Al día siguiente de la sesión recibí un mail de Violeta en el que me enviaba sus respuestas a las preguntas. Como era habitual en ella, no se atenía a la consigna, sino que respondía sólo algunas de las preguntas, sin escribirlas a su vez, por lo que había que inferir a cuál de ellas estaba respondiendo, y se entregaba a relatar otros episodios, en forma desordenada, retomando, o no, lo hablado en sesión.

Por este motivo, incluiré sólo aquella parte de su escrito en la que se focaliza directamente en las preguntas, o que guarda alguna relación con los temas presentes en ellas y en mis propias respuestas, ya que este material es el que resulta pertinente a los efectos de este trabajo. Para lograr mayor claridad en los comentarios posteriores, he agregado letras mayúsculas al comienzo de cada párrafo, para poder ubicarlo mejor.

“Hola.

A) Lo más importante de la sesión pasada es haberme dado cuenta el grado de desconexión que tengo cuando me sucede algo que me saca de contexto. Es como si me agarrara una amnesia completa.

(...) Fue importante porque no había notado en qué momentos me desconectaba, qué era lo que hacía que dejara de recordar el resto de las cosas que me van pasando.

Nunca pude darme cuenta qué era, qué me pasaba, dónde estaba mi mente, cómo me perdía en no sé qué pensamientos. Ahora tampoco lo tengo demasiado claro, pero al menos pude encontrar el punto. 
B) Al principio de la sesión, por momentos me sentí perdida, me costó, no sabía demasiado bien qué quería hablar. Después me relajé un poco y fue más fácil.

A veces, no sé bien por dónde empezar. Es como que necesito un empuje. Después me fui aflojando, dejando salir a quien realmente soy, me libero. Pero me cuesta en algunos momentos.

C) (...) Tu actitud me gusta, me siento bien, y me hace pensar, reflexionar.

Lo que me pasa es que soy muy buena escuchando, y pongo mucha atención en lo que me dices, me hace bien.

D) (...) Hablé de mucho de Manuel, que no sé hasta qué punto me hace tan bien, siempre quedo con un sabor amargo de la incertidumbre, incertidumbre. No sé dónde ubicarlo, ni dónde me ubica él a mí. Creo que con él me pasa que sé que no voy a llegar a nada y eso me hace sufrir, pero no puedo dejar de estar pendiente de sus whatsapps, de si me escribe o no, de si tiene ganas de verme.

(...) Otra de las cosas que me pasan es que tengo mucho miedo a estar sola y cuando estoy con alguien anhelo la soledad. Y pienso si estoy con la persona indicada o no, si realmente es lo que quiero o no, y ahí es donde soy infiel.

Esa es otra cosa que no puedo controlar a veces, es como un impulso sin frenos, sin remordimientos, sin culpa. Como si no fuera yo, como si me transformara en otra persona que actúa totalmente sin conciencia; y al otro día ni me acuerdo".

\section{Comparación entre los dos escritos y comentarios:}

Las consideraciones que realicé hasta el momento sobre mis propias respuestas tuvieron que ver, en lo esencial, con el tema de los indicadores, pero ahora se vuelve posible comparar las respuestas que por mi parte escribí, con los comentarios de Violeta.

Lo que resulta elocuente desde el comienzo es la concordancia en la importancia asignada al tema de la desconexión, lo que muestra que en este punto pude aprehender con claridad lo que para ella había resultado más importante de la sesión. Como suele suceder, Violeta agregó algunas precisiones en su escrito, que complementan lo hablado en la sesión: "Nunca pude darme cuenta qué era, qué me pasaba, dónde estaba mi mente, cómo me perdía en no sé qué pensamientos. Ahora tampoco lo tengo demasiado claro, pero al menos pude encontrar el punto" 
Otra concordancia se refiere a mi observación de que Violeta había entrado a la sesión en estado de desconexión y a medida que ésta transcurría, iba saliendo de dicho estado, se conectaba y se involucraba más.

Ella lo formula así: "Al principio de la sesión, por momentos me sentí perdida, me costó, no sabía demasiado bien qué quería hablar. Después me relajé un poco y fue más fácil".

Por mi parte, comento también que para ella fue importante que yo le hablara de su dependencia con los hombres. Si bien Violeta hace referencia a ese tema en D) ("no puedo dejar de estar pendiente de sus whatsapps, de si me escribe o no, de si tiene ganas de verme"), no queda claro si para ella fue uno de los temas más importantes de la sesión, tal como había sido mi impresión.

Tampoco queda claro si para ella fue un momento significativo cuando mencionó su miedo a preguntarle a Manuel si quería casarse con ella, tal como conjeturo yo. Pero el que mencione que no sabe dónde la ubica y que sufre pensando que no van a llegar a nada, concuerda, al menos temáticamente, con mi conjetura.

Respecto a lo que yo pongo en respuesta a la pregunta b), que se sintió entendida y acompañada, ella dice, en C) "Tu actitud me gusta, me siento bien, y me hace pensar, reflexionar. Lo que me pasa es que soy muy buena escuchando, y pongo mucha atención en lo que me dices, me hace bien".

Vemos en este caso una concordancia parcial, ya que ella pone el acento en su posición activa de pensar y reflexionar, mientras que yo lo pongo en mi actitud activa de acompañarla. Sin duda que ambas afirmaciones no se contraponen, pero hay una diferencia de acento que muestra que yo no capté adecuadamente su posición activa y reflexiva, que posee la mayor importancia en vistas a sus estados de desconexión, su impulsividad, etc.

Por último, mi conjetura de que la intervención que le resultó menos útil fue mi pregunta referida al vacío, no puede ser corroborada porque Violeta no hace la menor referencia a la misma.

Las consideraciones acerca de la comparación entre los dos escritos muestran la utilidad del método para monitorear la capacidad mentalizadora del terapeuta, en relación a un paciente concreto, en una sesión específica.

En este caso considero que puedo evaluar mi desempeño como satisfactorio, con un núcleo de aprehensión adecuada (el tema de la desconexión), una captación adecuada de su cambio a lo largo de la sesión, una concordancia parcial en el tema de cómo se sintió conmigo y una concordancia temática en el tema de Manuel. 
Por otro lado, cabe señalar también que el escrito de Violeta me permitió tomar conciencia de que no había captado adecuadamente su posición activa y reflexiva, lo cual no es una falla menor por mi parte, habida cuenta de las características de Violeta en lo que hace a sus procesos mentales. Sin su texto, sin la posibilidad de tener su propio punto de vista, no hubiera podido anoticiarme de este hecho. Gracias a este conocimiento, pude tener en cuenta esta capacidad suya en mayor medida, desde esa sesión en adelante.

La falta de información por parte de Violeta respecto a otros puntos, dificulta la contrastación de otras afirmaciones que yo había realizado en mis respuestas a las preguntas.

\section{Consideraciones finales:}

Llegados a este punto, cabe plantear al menos dos reparos al método propuesto, y un interrogante.

El primero de los reparos consiste en que para la puesta en juego de la tercera parte se hace necesario proponerle a los pacientes que lleven un diario de sesiones, lo cual acarrea no pocas dificultades. Una de ellas es que muchos pacientes no se sienten inclinados a hacerlo, o lo viven como una obligación, en cuyo caso se desvirtúa la utilidad que puede tener para ellos y todo el proceso puede llegar a complicarse. La introducción de esta variable, "extraña" al proceso habitual, debe ser hecha con muchas precauciones y estando muy atentos a la forma en que lo toma el paciente y al uso que se le da posteriormente a lo que él escribió (si se lo incluye, o no, en la sesión siguiente, por ejemplo). He realizado una serie de sugerencias en tal sentido, en otro lugar (Lanza Castelli, 2010)

Por otro lado, no son muchos los terapeutas que están dispuestos a instrumentar este procedimiento, con el que en general no tienen experiencia y que les produce muchas veces marcada incertidumbre respecto a cómo incluirlo en la dinámica para ellos conocida y habitual del tratamiento, tal como suelen llevarlo a cabo.

Una opción ante este reparo es que sea solamente el terapeuta quien responda a las preguntas, sin proponerle al paciente que lo haga. Esto es, que lleve a cabo sólo las partes 1 y 2 del método.

Es verdad que de este modo se torna imposible toda contrastación, pero si releemos con detenimiento lo escrito más arriba (después de consignar mis propias respuestas a las preguntas que me planteaba), veremos que se divide en tres partes: las dos primeras aluden a los beneficios que obtuve al plantearme las preguntas y responderlas. La tercera a la utilidad que obtuve al realizar la contrastación con lo escrito por la paciente. 
Los beneficios obtenidos en la primera y segunda parte del método, pueden sintetizarse de la siguiente manera:

a) Traducir lo implícito en explícito, lo que implica transformar una experiencia vivida en el modo del self prerreflexivo, en una serie de reflexiones sobre los propios procesos mentales y las intervenciones realizadas.

En otro lugar he mostrado -con cierto detalle- cómo el poner en palabras la experiencia vivida, incrementa la mentalización (Lanza Castelli, 2010b).

b) Identificar y denominar sensaciones, sentimientos, pensamientos fugaces o apenas esbozados que surcaron la mente de un modo inadvertido durante la sesión, de los que sólo nos enteramos en la práctica de responder las preguntas, y que de no ser por ello se habrían vuelto inaccesibles.

c) La contrastación entre las respuestas de la parte 1 y la parte 2 resulta de utilidad para identificar el grado en que podemos diferenciar nuestra mente de la del paciente, esto es, el grado en que podemos, o no, descentrarnos. La práctica reiterada del método favorece el incremento de la diferenciación mencionada.

d) Al escribir las respuestas suelen surgir ocurrencias involuntarias que son útiles para el autoanálisis y para la mejor comprensión de la contratransferencia (en el ejemplo clínico, mi pensamiento acerca de que es una mujer superficial). Asimismo, es habitual que surjan otras ocurrencias que permiten entender mejor lo ocurrido en sesión

e) Lograr una mayor aprehensión del modo en que funciona nuestra mente en la sesión. Así, la reflexión sobre lo ocurrido (respuesta a pregunta 2) me permitió tomar conciencia de la lectura preconsciente que había hecho de las actitudes corporales de Violeta, así como de mi reacción al respecto, cosas de las que no había sido consciente antes de ponerme a responder las preguntas. Este punto también habla de un incremento en la mentalización del self, de un incremento en la reflexividad y un pasaje de lo implícito a lo explícito.

f) Identificar fallas empáticas y explorar las razones de las mismas. En mi intervención en la sesión con Violeta detecto una falla empática (preguntarle por el vacío) y puedo rastrear los motivos de la misma, lo que me aclara un poco más la forma errónea en que puedo proceder cuando tengo un deseo activado. También en este punto, he incrementado la mentalización de mi mismo, en tanto pude inferir los estados mentales que se encontraban en la base de mi proceder.

Cabe aclarar que en este caso específico fueron éstos los beneficios, pero que en otros casos surgen otros de diferente índole. Lo que es común en todas las ocasiones es el incremento en 
el autoconocimiento y el aumento de la reflexividad, el descentramiento, la empatía y la comprensión del paciente.

Desearía agregar que por mi parte he realizado en numerosas ocasiones este procedimiento de responder las preguntas, sin que el paciente escriba a su vez, y en todos los casos he obtenido resultados equivalentes a los que acabo de enumerar.

Por último, una variante de este formato en que solamente el terapeuta escribe es compartir en supervisión o en un grupo de trabajo, una reconstrucción detallada de la sesión de que se trate y las respuestas que el terapeuta produjo a las preguntas en cuestión. La participación de los otros integrantes del grupo, sus preguntas y sugerencias, optimizan sin duda lo que el terapeuta podría lograr trabajando en soledad.

Por lo demás, este trabajo sobre las respuestas del terapeuta a las preguntas, puede convertirse en el eje de un taller que incluya también otros ejercicios vivenciales, lecturas de textos sobre mentalización, aplicados a casos clínicos y una serie de alternativas como las llevadas a cabo por Ensink y colaboradores (2013), por Haslam-Hopwood et al. (2006) en sus grupos de psicoeducación, por Bleiberg en su trabajo con profesionales en crisis (2006), por Williams et al. en su training para residentes en psiquiatría (2006), etc.

Retomando el tema de los reparos a la inclusión de la tercera parte, podríamos decir que si dichos reparos son removidos, se torna posible obtener -con la inclusión de ese sector del método- nuevos beneficios, que sintetizamos del siguiente modo:

a) En primer término, recibimos un valioso feedback respecto de cómo se ha sentido el paciente en sesión, cuáles de nuestras intervenciones le han resultado de utilidad y cuáles no, qué ha sido para él lo más importante de la sesión, etc.

Cabe referir que en los últimos años se han llevado a cabo una serie de investigaciones sobre la utilidad que el feedback por parte del paciente posee para el profesional.

Algunas de estas indagaciones se han centrado en el feedback que el terapeuta recibe acerca de las mejorías o empeoramientos que el paciente experimenta a medida que se desarrolla el tratamiento. En diversos estudios llevados a cabo por Whipple, Lambert, Vermeersch y colaboradores (Lambert et al, 2001), se dividió en dos grupos a un conjunto de pacientes que tenían inicialmente un pronóstico de resultado negativo o nulo. A todos ellos se les pidió que, tras cada sesión de psicoterapia llenasen un cuestionario por medio del cual se podía evaluar la mejoría o empeoramiento que experimentaban. Los terapeutas de uno de esos grupos recibían el feedback correspondiente, en base a la información de los cuestionarios. Los del grupo de control, en cambio, no la recibían. 
Los resultados mostraron que los terapeutas que recibían el feedback podían modificar el abordaje clínico que estaban llevando a cabo, con lo cual conseguían que los pacientes continuaran en el tratamiento, anticipándose con ello a una eventual deserción, y lograban, en un porcentaje considerable, revertir el estado de cosas problemático existente y conseguir resultados positivos. Los terapeutas que no recibían dicho feedback no hacían corrección alguna, con el consiguiente empeoramiento y deserción de un mayor número de pacientes que en el grupo anterior. De ahí la importancia que tiene el feedback que nos entrega el paciente, sea en la forma escrita que propongo en esta ocasión, sea en forma sistemática en el transcurso de las sesiones, tal como he sugerido en otro lugar (Lanza Castelli, 2013).

b) Gracias a la tercera parte del método podemos advertir las concordancias y divergencias entre nuestras conjeturas acerca de lo experimentado por el paciente y lo que éste efectivamente experimentó. A partir de esta comparación podemos evaluar con nitidez la calidad del funcionamiento de nuestro mentalizar dirigido al otro, lo cual implica, entre otras cosas, identificar cuán ajustada es la captación que tenemos de su experiencia, cuáles son los aspectos de la misma que no aprehendemos, o que aprehendemos en forma parcial, sesgada o distorsionada, en qué medida hacemos recaer sobre él proyecciones o atribuciones basadas en nuestros propios procesos y contenidos mentales y que perturban una más adecuada captación de su realidad psíquica, etc.

c) La indagación sobre las razones de las discrepancias nos puede ayudar a identificar los motivos de nuestras fallas y trabajar sobre los mismos. Este trabajo forma parte del método, aunque cabe señalar que tiene sus límites aquello que podemos elaborar por nuestra exclusiva cuenta. En algunas ocasiones puede ocurrir que el método nos permita identificar con claridad las fallas, pero que para la indagación y procesamiento de sus motivos necesitemos la ayuda ajena, ya sea en una supervisión o en un análisis personal.

d) El contraste entre nuestras conjeturas y las respuestas del paciente nos puede mostrar también aquello que no hemos percibido o valorado adecuadamente (como en mi caso, la posición activa de Violeta, que había subestimado), a los efectos de corregirlo y encauzar el tratamiento de un modo que resulte de mayor utilidad para el paciente.

Cabe agregar, no obstante, que ciertas discrepancias, por ejemplo respecto a lo que ha sido lo más importante de la sesión, pueden obedecer también a defensas del paciente que lo llevan a minimizar lo que para él sí tiene importancia. De todas formas, mediante el uso del método que estoy proponiendo buscaremos evaluar si hemos sido capaces de identificar ese funcionamiento defensivo en el transcurso de la sesión, de modo tal que podamos al menos intentar conjeturar -al escribir la parte 2- cómo dicho funcionamiento habrá de incidir en las 
respuestas del paciente a las preguntas que le formulamos. En este caso, la magnitud de la discrepancia dará cuenta del éxito, mayor o menor, que hemos tenido en dicho conjeturar.

El segundo reparo es de otra índole y consiste en que este método no ha sido corroborado por una evaluación de la capacidad de mentalizar previa a la utilización del mismo a lo largo de un período de tiempo, y una evaluación posterior que permita, al contrastarla con la primera, advertir de modo fehaciente si tal incremento ha tenido lugar, o si es sólo una ilusión de quien practica el método.

La única forma, en mi opinión, de zanjar este segundo reparo, es llevar a cabo una investigación rigurosa, con varios participantes, en los que se practiquen evaluaciones de la índole señalada. Hasta el momento esa investigación no ha sido realizada.

Por último, el interrogante al que hice referencia al comienzo de este apartado, es cuán generalizable es el incremento en el mentalizar que se logra con un paciente determinado, con el que se ha utilizado el método a lo largo de un tiempo razonable.

Mi propia experiencia, así como la de otros colegas que han utilizado el método que estoy proponiendo, muestra con toda elocuencia que el uso del mismo incrementa la capacidad de mentalizar al paciente con el que se lo está utilizando. Pero no hemos llevado a cabo una investigación controlada, que nos permita ver si dicho incremento se ha generalizado a los otros pacientes, con quienes no se utilizó el método, aunque la impresión subjetiva de mis colegas y mía es que tal generalización sí había tenido lugar.

Por otra parte, cabe agregar que nada impide que el método sea utilizado con más de un paciente a la vez, lo que agrega el beneficio de poder contrastar el propio desempeño con distintos pacientes, hecho que resulta de utilidad para identificar aquellos con los que tenemos mayores dificultades en nuestra empatía y comprensión y, por ejemplo, llevarlos a supervisión. Asimismo, esta ampliación propicia el incremento del campo de nuestra autoexploración en tanto nos puede eventualmente permitir constatar que ciertas características de algunos pacientes nos dificultan la captación de sus procesos internos, mientras que las de otros favorecen dicha captación. Este hecho puede llevarnos a revisar qué aspectos de nuestra interioridad son tocados por las características que nos producen dificultades, ahondando entonces en nuestro autoanálisis o en nuestro análisis personal.

No obstante, para quien desee comenzar a utilizar el método, mi sugerencia es que lo haga con un solo paciente y que lleve a cabo solamente las partes 1 y 2 , al menos hasta que se encuentre suficientemente familiarizado con el mismo y desee ampliarlo, sea utilizándolo con otros pacientes, sea incluyendo también la parte 3 , o ambas cosas. 
Concluyo formulando la esperanza de que el método propuesto ayude a distintos colegas, particularmente a aquellos que están haciendo su formación, pero no sólo a ellos, a trabajar sobre sí mismos a los efectos de incrementar su capacidad de mentalizar (tanto respecto al self como respecto al otro) y, de este modo, obtener mejores resultados en su práctica clínica, independientemente de su marco teórico de referencia.

\section{REFERENCIAS}

Allen, J., Fonagy, P., Bateman, A. (2008) Mentalizing in Clinical Practice.Arlington: American Psychiatric Publishing, Inc.

Baldwin, S. A., \& Imel, Z. E. (2013). Therapist effects: Findings and methods. En M. J. Lambert (Ed.), Bergin and Garfield's handbook of psychotherapy and behavior change (6th ed., pp. 415-456). Hokoben: John Wiley \& Sons.

Bateman, A., Fonagy, P. (2016) Mentalization-Based Treatment for Personality Disorders A Practical Guide. Oxford: Oxford University Press.

Bion, W.R. (1970) Atención e interpretación. Buenos Aires: Paidos, 1974

Bleiberg, E. (2006) Treating Professionals in Crisis: A Mentalization-Based Specialized Inpatient Program, en Allen. G.J., Fonagy, P. (eds.) (2006) Handbook of Mentalization-Based Treatment. West Sussex: John Wiley \& Sons Ltd.

Cologon, J., Schweitzer, R.D., King, R., Nolte, T. (2017) Therapist Reflective Functioning, Therapist Attachment Style and Therapist Effectiveness" Springer Science + Business Media. New York 1917

Ensink, K., Maheux, J., Normandin, L., Sabourin, S., Diguer, L., Berthelot, N, et al. (2013). The impact of mentalization training on the reflective function of novice therapists: A randomized controlled trial. Psychotherapy Research, 23(5), 526-538.

Fonagy, P., Target, M. (1997) Attachment and reflective function: Their role in self Organization. Development and Psychopathology, 9 (1997), 679-700

Fonagy et al. (1998) Reflective Functioning Manual. Unpublished

Fonagy, P; Luyten, P (2009) A developmental, mentalization-based approach to the understanding and treatment of borderline personality disorder. Development and Psychopathology 21, 13551381

Fonagy, P; Luyten, P (2010) Mentalization: Understanding Borderline Personality Disorder, en Fuchs, Sattel, Henningsen (eds) The Embodied Self. Dimensions, Coherence and Disorders. Schattauer.

Fonagy, P, Gergely, G, Jurist, E, Target, M (2002) Affect Regulation, Mentalization, and the Development of the Self. New York: Other Press.

Haslam-Hopwood, T.G:, Allen, J.G., Stein, A., Bleiberg, E. (2006) Enhancing Mentalizing through Psycho-Education. En Allen, J.G., Fonagy, P. (eds) Handbook of Mentalization-Based Treatment. Chichester: John Wiley \& Sons Ltd.

Lambert, M. J. (2013). The efficacy and effectiveness of psychotherapy. En M. J. Lambert (Ed.), Bergin and Garfield's handbook of psychotherapy and behavior change (6th ed., pp. 274-351). Hokoben: John Wiley \& Sons. 
Lambert, M. J., Whipple, J. L., Smart, D.W., Vermeersch, D, A., Nielsen, S.L.,Hawkins, E.J. (2001) The Effects of Providing Therapists With Feedback On Patient Progress During Psychotherapy: Are Outcomes Enhanced? Psychotherapy Research 11 (1) 49-68

Lanza Castelli, G. (2008). El feedback al terapeuta y la escritura del "diario de sesiones". Revista de la Asociación de Psicoterapia de la República Argentina, 1(1)

Lanza Castelli, G. (2010a) (comp.) La escritura como herramienta en la psicoterapia. Madrid: Psimática.

Lanza Castelli, G. (2010b) Poner en palabras, mentalización y psicoterapia. Aperturas Psicoanalíticas. Revista Internacional de Psicoanálisis, Nro 36

Lanza Castelli, G. (2013) La mentalización y el feedback del paciente en el intercambio clínico. Revista Diagnosis, Nro 10 http://revistadiagnosis.org/prosam/10/comentarios-de-libros/item/25diagnosis-n\%C2\%Bo10-2012

Lanza Castelli, G., Bilbao Bilbao, I. (2012) Un método para la Evaluación de la Mentalización en el Contexto Interpersonal. EFPP Psychoanalityc Psychotherapy Review (Revista por Internet http://www.efpp.org/review/EFPP_review_02_2012.pdf)

Rennie, D. L. (1992 ). Qualitative analysis of the client's experience of psychotherapy: The unfolding of reflexivity . In S. Toukmanian \& D. Rennie (Eds.), Psychotherapy process research: Paradigmatic and narrative approaches (pp. 211-233). Newbury Park, CA : Sage.

Rennie, D. L. (1994). Clients' deference in psychotherapy. Journal of Counseling Psychology , 41(4), 427-437.

Rennie, D. L. ( 2001 ). The client as self-aware agent in counselling and psychotherapy. Counselling and Psychotherapy Research , 1 ( 2 ), $82-89$.

Original recibido con fecha: 15/09/2017 Revisado: 27/09/2017 Aceptado: 30/09/2017

NOTAS:

' Gustavo Lanza Castelli es autor de más de 70 trabajos, publicados en revistas nacionales y extranjeras, sobre psicopatología, análisis del discurso, escritura y psicoterapia, mentalización y psicoterapia. Es compilador y coautor del libro La escritura como herramienta en la psicoterapia, en coautoría con Héctor Fernández Alvarez y Héctor Fiorini, editado en 2011 por Psimática (España). Ha diseñado dos pruebas para evaluar la mentalización: a) El Método para la Evaluación de la Mentalización en el Contexto Interpersonal (MEMCI) (junto con Itziar Bilbao Bilbao) publicado en la Psychoanalytic Psychotherapy Review, European Federation for Psychoanalytic Psychotherapy. http://www.efpp.org/. Se encuentra validado; b) El Test de Situaciones para la Evaluación de la Mentalización (TESEM).Publicado en la Revista de la Asociación de Psicoterapia de la República Argentina. Año IV, Nro 2 http://www.revistadeapra.org.ar/ultimo.htm Se encuentra en proceso de validación. expresa. Este material es para uso científico y profesional exclusivamente y puede contener información clínica sensible. Los editores no se responsabilizan de los contenidos de los autores. Dirigir las consultas sobre derechos y autorizaciones a ceir@psicoterapiarelacional.es 